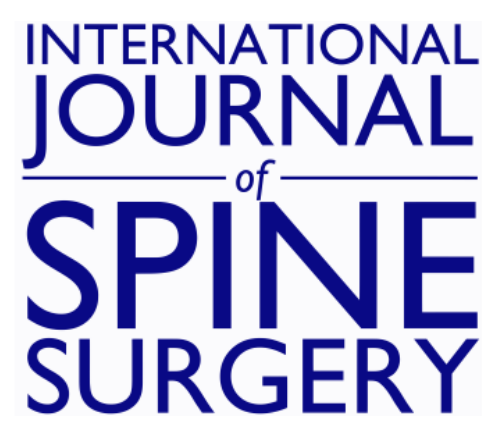

\title{
Etiology and Management of Spinal Deformity in Patients With Parkinson's Disease
}

\author{
ROY RUTTIMAN, ADAM E. M. ELTORAI and ALAN H. DANIELS
}

Int J Spine Surg 2018, 12 (1) 15-21

doi: https://doi.org/10.14444/5003

http://ijssurgery.com/content/12/1/15

This information is current as of April 26, 2023.

Email Alerts Receive free email-alerts when new articles cite this article. Sign up at: http://ijssurgery.com/alerts 


\title{
Etiology and Management of Spinal Deformity in Patients With Parkinson's Disease
}

\author{
ROY RUTTIMAN, MS, ${ }^{1}$ ADAM E. M. ELTORAI, BA, ${ }^{1}$ ALAN H. DANIELS, MD ${ }^{2}$ \\ ${ }^{1}$ Warren Alpert Medical School of Brown University, Providence, Rhode Island, ${ }^{2}$ Division of Spine Surgery, Department of Orthopaedic Surgery, Warren Alpert \\ Medical School of Brown University, Providence, Rhode Island
}

\begin{abstract}
Patients with Parkinson's disease (PD) commonly develop severe spinal deformity, including scoliosis, antecollis, camptocormia, and Pisa syndrome. The etiology of PD-associated spinal deformity is not completely understood and in most cases is likely due to multiple interrelated factors, including central dystonia and focal myopathy. Once spinal deformity has occurred, surgery is often the only modality that can correct the condition, although control of the movement disorder through medication and deep brain stimulation may slow progression. Advances in spinal instrumentation and deformity correction techniques have improved the outcomes of PD spinal deformity patients, though complications and revision surgery rates remain high. Surgical intervention is reserved for individuals who are physiologically healthy and whose condition is refractory to nonoperative management and follows similar treatment principles as other causes of neuromuscular scoliosis/kyphosis. Spinal deformity patients with PD are optimally treated by spinal deformity surgeons who are familiar with the unique needs of PD patients, with vigilant preoperative and postoperative treatment of their movement disorder and bone density.
\end{abstract}

Lumbar Spine

Keywords: spinal deformity, Parkinson's disease, scoliosis, antecollis, camptocormia, Pisa syndrome

\section{INTRODUCTION}

Parkinson's disease (PD) is a degenerative movement disorder of the central nervous system, characterized by tremor, rigidity, and akinesia. Additionally, PD patients often develop stereotypical postural changes, including stooped posture, dropped head, and a flexed trunk, hips, and knees. ${ }^{1-4}$ In some patients, postural changes manifest as more disabling spinal deformities, such as scoliosis, antecollis, camptocormia, and Pisa syndrome. Abnormalities of spinal alignment shift the patient's center of gravity, forcing abnormal spinal load bearing, which in turn, may accelerate normal and age-related spinal degeneration. ${ }^{5,6}$ These biomechanical and neurophysiological changes place PD patients at increased risk for spinal deformity and other spinal conditions. ${ }^{7}$ As PD and spinal conditions typically affect older individuals, the prevalence of PDassociated spinal deformity is projected to increase with the aging population. ${ }^{1,3,5-7}$

\section{PATHOPHYSIOLOGICAL THEORIES}

The underlying cause of spinal disorders in PD remains controversial. ${ }^{5,8,9}$ Possible contributing factors include muscle rigidity, proprioceptive disintegration, medication effects, and soft tissue changes. ${ }^{2,5}$ Dystonia and myopathy have also been explored as causal origins of spinal deformity. Supporters of the dystonia theory argue that postural disorders-particularly the characteristic stooped posture - arise from abnormal flexion of the paraspinal and truncal muscles. ${ }^{5,8}$ Such abnormal muscular contractions have been clinically observed in patients with PD presenting with early antecollis and camptocormia. ${ }^{8}$

The involvement of dystonia may be suggested by electromyography (EMG) studies. However, Di Matteo et al. ${ }^{10}$ found characteristic dystonic activity in the paraspinal muscles in just 3 of 10 patients with PD presenting with Pisa syndrome (lateral spinal tilt). Overall, EMG studies on paraspinal muscles in patients with PD are inconsistent. ${ }^{8-12}$ Electromyography recordings in patients with $\mathrm{PD}$ can be difficult for researchers and painful to subjects, resulting in imprecise measurements and suboptimal studies. 8,13

Focal myositis, myasthenia gravis, and other primary myopathies have been associated with postural-induced spinal deformity. ${ }^{8,14-16}$ Electromy- 


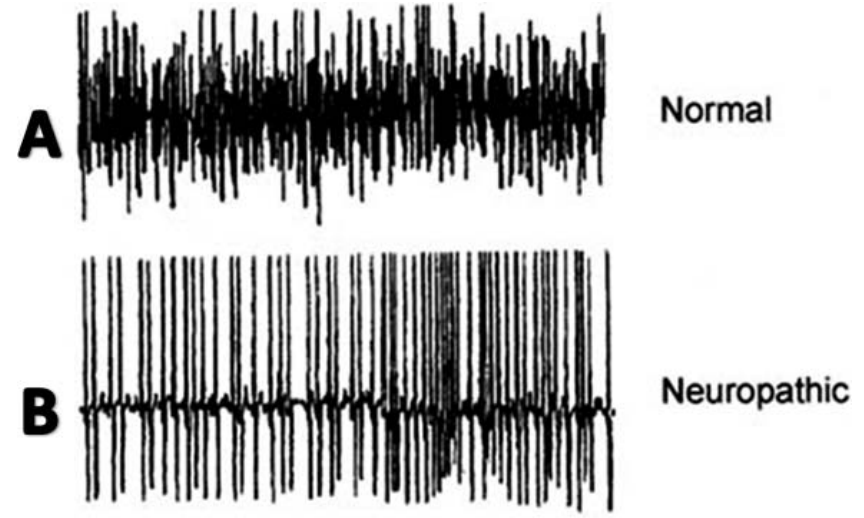

\section{Propathic}

Figure 1. (A) Normal summation of motor unit action potential pattern (MUAP). (B) Classic neuropathic MUAP with large deflections from baseline. (C) Characteristic short deflections of myopathic MUAP. ${ }^{16-18}$

ography, muscle imaging, and histological studies serve as the primary evidence for the myopathy theory. Electromyography studies (Figure 1) on truncal muscles in patients with PD have revealed myopathic patterns (ie, fibrillation potentials and small polyphasic motor unit potentials), ${ }^{16-18}$ though the reproducibility of EMG findings is poor. Computed tomography studies in patients with PD demonstrate muscle atrophy and nonspecific hypodense findings restricted to paraspinal muscles. ${ }^{8}$ Histological studies on paraspinal muscle biopsies from patients with PD have shown myopathic changes including ragged red fibers, myofibrillar disorganization, nuclear polymorphism, cellular atrophy, and proliferation in extramuscular connective tissue. ${ }^{8,16,19,20}$ Opponents argue that these histological features can also be found in agematched patients with neither PD nor spinal deformity. 8,10

Dystonia and myopathy may both play a role in the pathophysiology of PD-associated spinal deformity. As paraspinal dystonia is more readily observed in early-stage and young-onset $\mathrm{PD}$, some investigators consider dystonia to be an initial yet transient feature in the development of spinal deformity. ${ }^{8}$ With advanced-stage PD, typical dystonic features are progressively replaced by focal myopathy, characterized by pronounced muscular changes and spinal deformity.

Select cases may shed light on the pathophysiology of PD-associated spinal deformity. Uzawa et al. $^{20}$ reported cases in which dopamine agonists caused or worsened Pisa syndrome or antecollis in

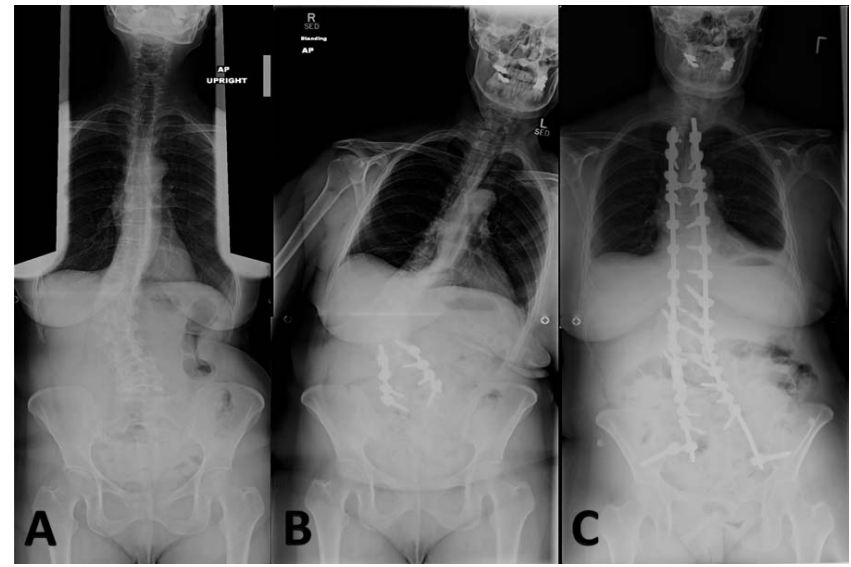

Figure 2. Parkinson's disease patient with scoliosis (A): underwent L2-5 decompression and fusion (B), leading to curve decompensation and progression requiring T2-pelvis posterior spinal arthrodesis (C).

PD patients within weeks to months of treatment. Fortunately, medication-induced spinal deformity corrected upon discontinuing treatment. Neurotransmitter imbalances secondary to medications may dysregulate axial muscle tone, contributing to spinal changes. ${ }^{20}$ The majority of individuals on dopamine agonists do not acquire spinal deformity, yet these case reports provide insight into the mechanisms of spinal changes in PD.

Soft-tissue changes are a potential pathophysiological mechanism in the development of skeletal deformities. ${ }^{14}$ Ashour et al. ${ }^{14}$ described alterations in soft tissue, particularly connective tissue fibrosis and muscle atrophy and subsequent fixed contracture development, as the possible underlying mechanism of limb deformities seen in PD patients, which may also contribute to spinal deformity. Further research is needed to assess whether such soft-tissue changes may also be at the root of axial skeletal deformities.

\section{COMMON SPINAL DEFORMITY PATTERNS IN PARKINSON'S DISEASE}

Patients with PD may develop severe deforming conditions, including scoliosis, camptocormia, antecollis, and Pisa syndrome..$^{2,4,5,14}$

\section{Scoliosis}

Scoliosis is coronal curvature of the spine, frequently coupled with vertebral rotation (Figure 2). ${ }^{5,21-24} \mathrm{X}$-ray imaging is diagnostic and reveals coronal plane spinal curvature $>10^{\circ} .{ }^{5,21,23}$ Parkinson's disease scoliosis is often associated with a reduction in physiological lordosis, lateral listhesis, 


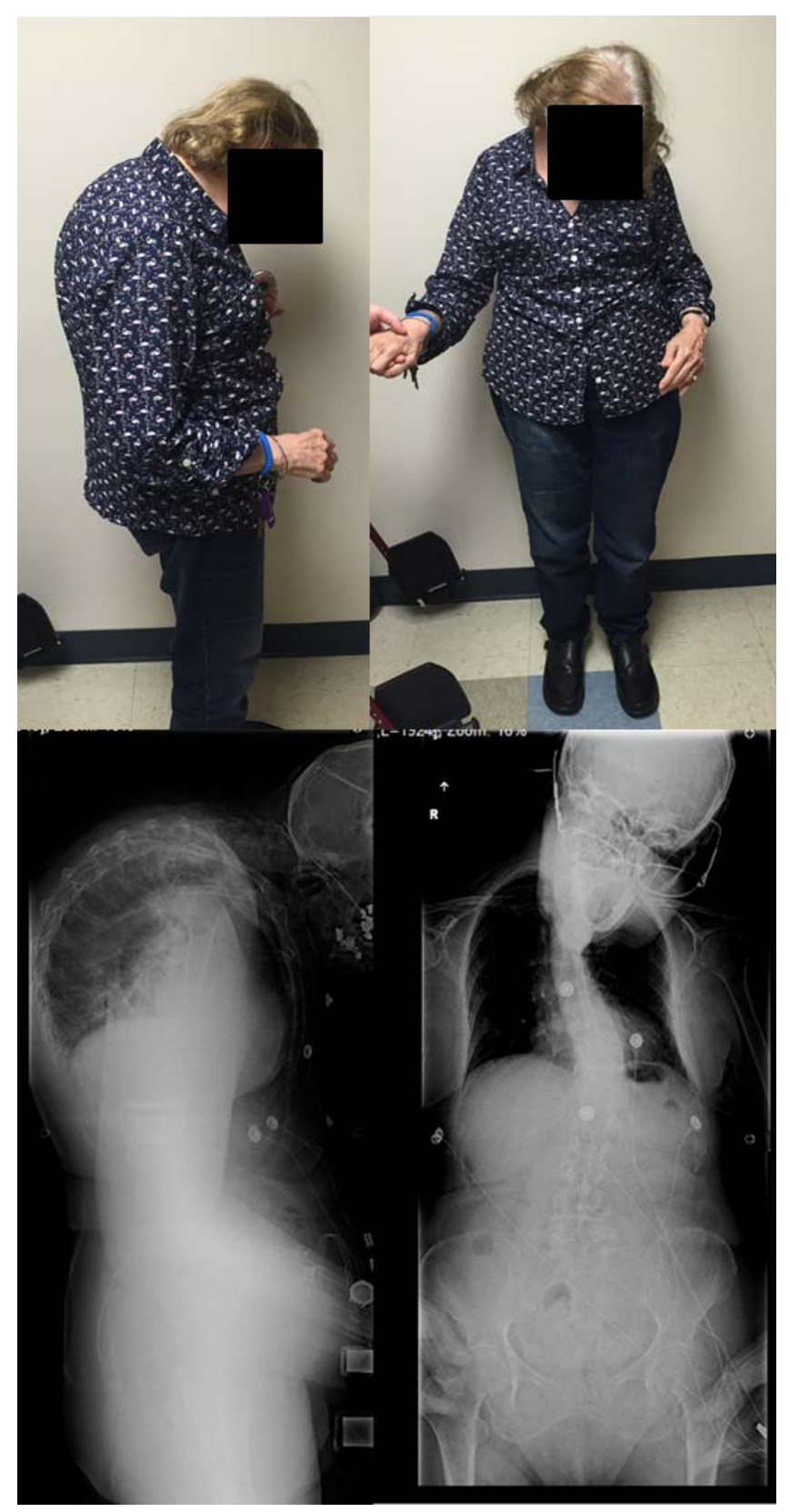

Figure 3. Parkinson's disease patient with antecollis.

and spondylolisthesis. ${ }^{21}$ Patients with PD are more likely to develop scoliosis than the general elderly population. ${ }^{5,24}$ The prevalence of scoliosis in patients with PD ranges from $8.5-60 \%$ as compared to $6-32 \%$ in healthy age-matched individuals. ${ }^{5}$

Surgical intervention to correct scoliosis in patients with PD is often reserved for patients with concomitant myelopathy or radiculopathy or severe sagittal or coronal malalignment. ${ }^{1-5,25}$ Short-segment decompression and fusion can be considered in select symptomatic, highly motivated patients, although the risk of progressive deformity should be discussed preoperatively. ${ }^{7}$ Postoperative spinal deformity progression following short-segment lumbar surgery has been reported, which frequently requires revision surgery. ${ }^{1,7}$

\section{Antecollis}

Antecollis is a forward flexion of the head and neck-commonly referred to as dropped head syndrome - coupled with increased axial tone (Figure 3). As the deformity progresses, patients with antecollis display markedly reduced range of motion and eventually develop a fixed deformity. ${ }^{26-28}$ The occurrence of antecollis is most common in patients with PD or peripheral neuromuscular disorder, such as multiple system atrophy. ${ }^{2,14}$ Approximately 6\% of PD patients develop antecollis. There is mixed evidence supporting use of levodopa, clonazepam, botulinum toxin injections, and deep brain stimulation for antecollis in PD. ${ }^{2,5,26-29}$

Although studies evaluating vertebral fusion to treat antecollis are limited, ${ }^{1,30}$ surgical treatment may benefit individuals with poor intake or difficulties breathing. ${ }^{26-28}$ Furthermore, weakened upper back muscles and extensor muscles of the neck seen in antecollis may increase the risk of instrumentation failure at the lower instrumented vertebra $;^{5}$ thus, many surgeons advocate extending the fusion to the low thoracic or upper lumbar spine. Spinal osteotomy may be required if the deformity is fixed.

\section{Camptocormia}

Known as bent spine syndrome, camptocormia is described as pronounced forward flexion of the thoracolumbar spine, typically characterized by $\geq 45^{\circ}$ flexion when standing (Figure 4)..$^{2,8,11-14}$ Early camptocormia often corrects when the inflicted patient is supine, although this deformity may become fixed when long standing. ${ }^{11}$ The prevalence of camptocormia in PD patients is between 3 and $18 \%{ }^{5,14}$ Camptocormia typically presents 5-10 years after the onset of PD, affects older patients, and is associated with more severe PD. ${ }^{2,5,8,12}$

Various treatment strategies have yielded equivocal results for camptocormia in PD patients, including braces levodopa, botulinum toxin injections, and deep brain stimulation. ${ }^{11,19,31-33}$

Spinal surgery is a beneficial treatment option for camptocormia. ${ }^{5,11-13}$ Optimization of postoperative sagittal balance is mandatory to prevent proximal junctional kyphosis. ${ }^{1,5,7}$ Many patients with PDassociated camptocormia have kyphotic failure at 


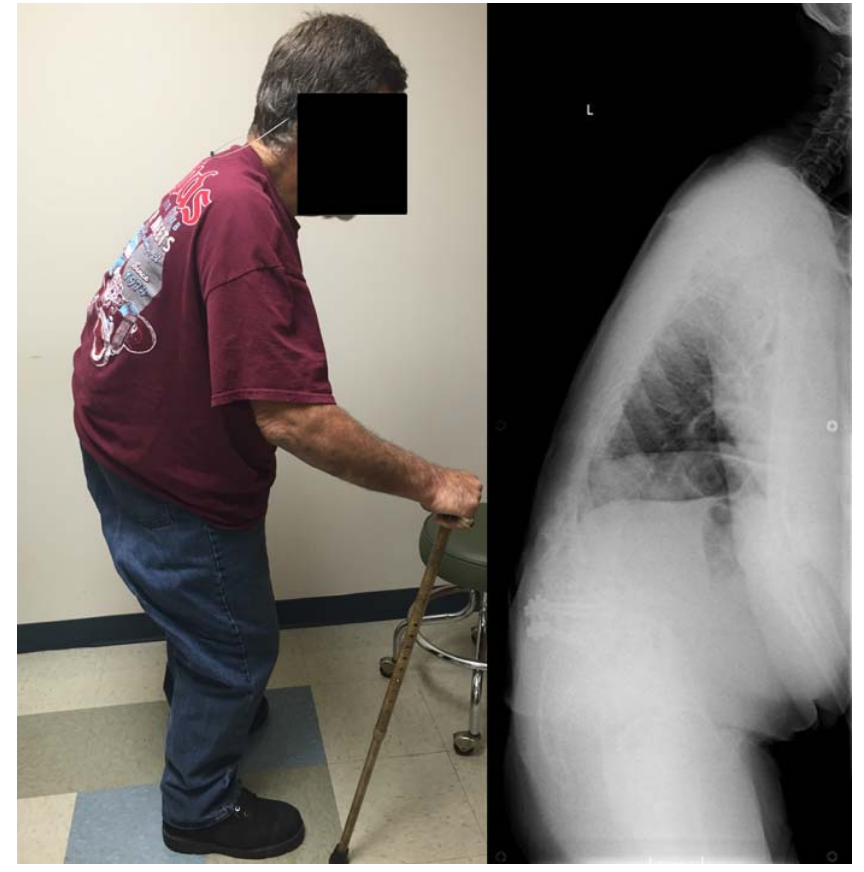

Figure 4. Parkinson's disease patient with Camptocormia

the cephalad junction of the deformity and eventually undergo upper cervical or even occiput to ilium fusions. ${ }^{1,12}$

\section{Pisa Syndrome}

Pisa syndrome is characterized by $>15^{\circ 5,18}$ of lateral truncal flexion when sitting or standing, which often resolves with passive mobilization or supine positioning (Figure 5). Affecting 2\% of PD patients, ${ }^{5}$ Pisa syndrome has been described as a truncal dystonia, ${ }^{18,34}$ a cholinergic-dopaminergic imbalance, ${ }^{2,35-37}$ and as a possible precursor to scoliosis in PD. ${ }^{2,5,37}$ As the deformity evolves, patients are prone to falling and may experience pain from rib-pelvis abutment. ${ }^{10}$

Modification of dopaminergic therapy has been shown to be an effective treatment option in some cases of early-stage Pisa syndrome. ${ }^{38}$ Anticholinergics, botulinum toxin injections, and deep brain stimulation treatment strategies have demonstrated mixed results. ${ }^{35,37}$ Like scoliosis, Pisa syndrome patients with severe coronal plane deformity or concomitant myelopathy or radiculopathy may benefit from long-segment spinal arthrodesis with correction of the deformity. ${ }^{1,3,5}$

\section{MANAGEMENT CONSIDERATIONS}

The management of spine deformities patients with PD requires careful consideration, surgical

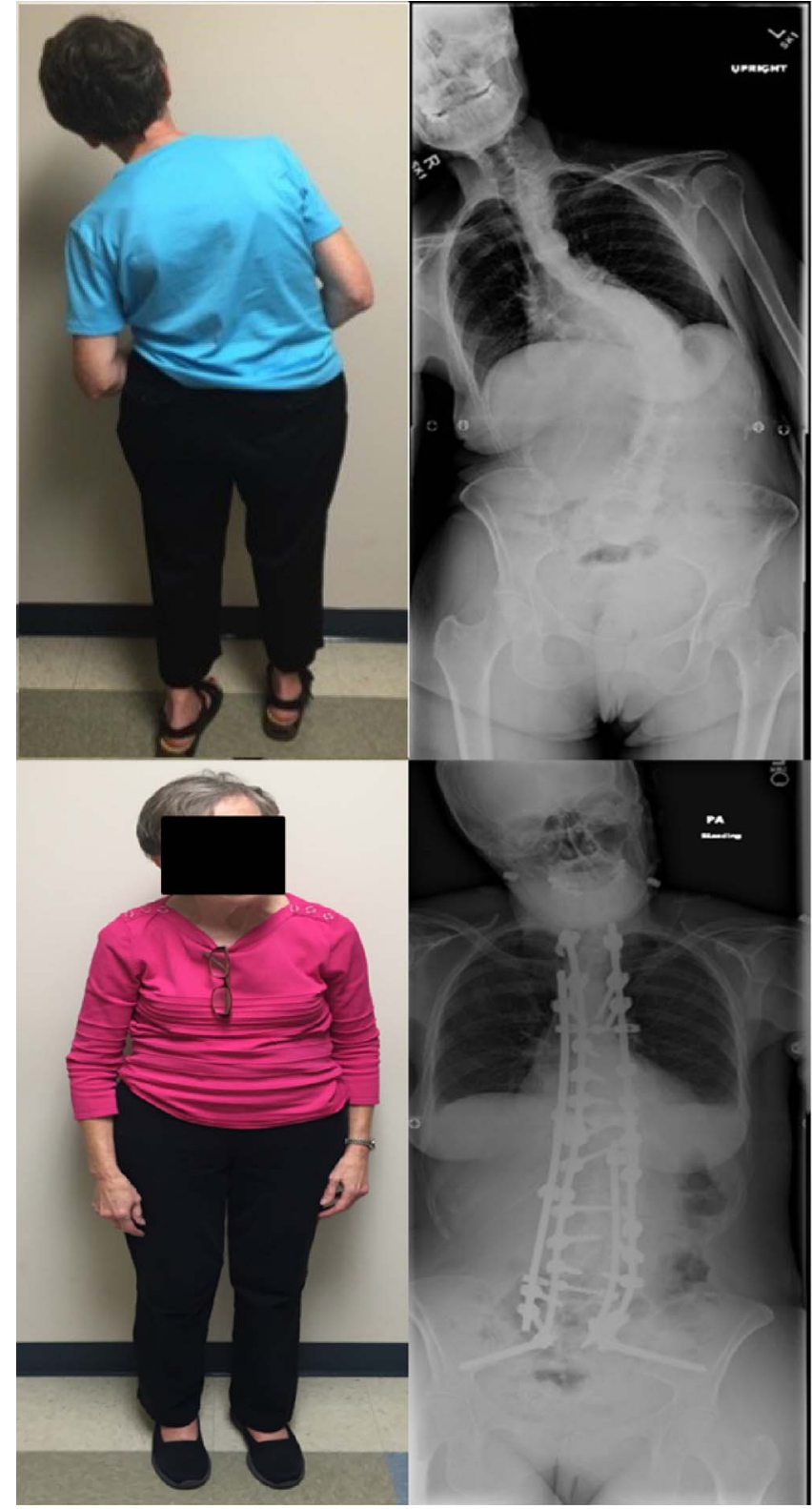

Figure 5. Parkinson's disease patient with Pisa syndrome.

planning, and instrumentation with robust spinal fixation due to common mechanical instrumentation failure and junctional kyphosis. Patients with PD commonly possess decreased bone mineral density, weakened paraspinal and truncal muscular strength, and concomitant medical problems., ${ }^{7,39}$ Preoperatively, Parkinsonian symptoms should be carefully managed with medication or deep brain stimulation, and bone mineral density should be optimized. If spine surgery is indicated, preoperative assessment must account for the biomechanical challenges associated with PD.,39 The patient's functional level, medication regimen, life goals, and 
natural progression of PD should all inform the development of a patient-specific treatment plan.

Treatment of spinal disorders should be focused on decreasing pain and preserving function by optimizing spinal alignment. While medication, activity modification, and physiotherapy may serve as viable treatment options for most patients with PD, surgery may be recommended for patients with refractory disease. ${ }^{1-4,7,39}$

As with spinal deformities due to other neuromuscular diseases, surgical treatment of spinal deformity in PD differs from that of degenerative and idiopathic scoliosis. Spinal deformity in PD may exhibit characteristic fixed lumbar sagittal and coronal deformity with little associated rotational deformity, as opposed to idiopathic scoliosis, which is characterized by axial rotational deformity. ${ }^{3,4}$ In PD patients, prevention of spinal deformity progression and preservation of spinopelvic balance may be achieved by spinal fusion from the thoracic spine to the pelvis, with an emphasis on reestablishing physiological lumbar lordosis and proper global sagittal alignment. $3,4,7$

Surgeons who are experienced with PD patients produce better outcomes compared to those who are unfamiliar with the unique needs of PD patients. ${ }^{7}$ Careful preoperative planning and surgical execution are necessary to optimize sagittal balance and prevent junctional failure. ${ }^{12,39}$ Utilizing additional outrigger rods is also recommended to prevent rod fracture. ${ }^{40}$ Communication between the patient's spine surgeon and neurologist is recommended before surgery as well for optimal control of the movement disorder.

\section{SURGICAL OUTCOMES}

Parkinson's disease patients with spinal deformity are prone to intraoperative and postoperative complications, with many patents requiring revision surgery. ${ }^{1,4,7,39}$ Postoperative infection $(17 \%)$ and instrumentation failure and/or pull out $(35 \%)$ accounted for the majority of surgical complications in a study performed by Koller et al., ${ }^{7}$ with $33 \%$ of all patients necessitating revision surgery. Complications were linked to pseudoarthrosis and junctional kyphosis, while surgical success was associated with reconstruction of sagittal balance. ${ }^{7}$

Some series have demonstrated very poor outcomes following treatment of PD-associated spinal deformity. In a 14-patient PD study by Babat et al., ${ }^{1}$
$86 \%$ (12 patients) of PD patients who underwent spinal surgery required additional surgery, with the majority of patients $(76 \%)$ requiring surgeries at the same or adjacent level to address instability. Two patients $(14 \%)$ experienced wound infections during the course of treatment. Babat et al. ${ }^{1}$ recognized kyphosis or segmental instability at the operative site or adjacent levels as the principle mechanism of failure. Similarly, Sapkas et al. ${ }^{4}$ showed that 5 of the 10 PD patients involved in their study required reoperation. Of the patients that did not need additional surgery, two developed camptocormia, one developed flatback syndrome, one developed proximal junctional kyphosis, and one was lost to follow up. ${ }^{4}$

Despite some poor outcomes, Koller et al. $^{7}$ found that the majority of patients with PD $(78 \%)$ who underwent spinal surgery for spinal deformities were satisfied with their clinical outcomes, whereas only $22 \%$ of patients were either not satisfied or uncertain of their outcomes. Patient satisfaction and good outcomes were connected with patients who received optimized medical treatment by a neurologist prior to surgery. ${ }^{25}$ Moreover, physical therapy after surgery and family involvement in healthcare delivery via discussions regarding long-term care and foreseeable complications were also associated with patient satisfaction. ${ }^{25}$

The number of days in hospital care for patients with PD is greater than for the general population who underwent the same spinal procedures. ${ }^{5,24,25}$ Several factors may contribute to poor outcomes in PD patients, including delayed mobility, longer hospitalization time, reduced mobility, and other comorbidities. As such, close monitoring of potential complications and early initiation of rehabilitation is even more important in PD patients than it is in the general population., ${ }^{4,25}$

\section{CONCLUSION}

Patients with PD often develop spinal deformity, with scoliosis, antecollis, Pisa syndrome, and camptocormia accounting for the most prevalent disorders within the population. While the etiology of spinal deformity in PD remains unknown, it is most likely attributable to multiple interrelated factors. Surgical management is multifaceted and requires a patient-specific approach that considers the patient's functional status and responsiveness to medication. Furthermore, the relationship be- 
tween the spinal deformity and the patient's symptomatology must be carefully assessed before committing to surgical intervention. Adequate bone density alongside management of nonspinal PD symptoms should be pursued preoperatively. Positive postoperative outcomes following spinal deformity correction are possible through optimized preoperative and postoperative care, familiarity with unique PD challenges during surgery, meticulous surgical execution, and careful patient selection.

\section{REFERENCES}

1. Babat LB, McLain RF, Bingaman W, Kalfas I, Young $\mathrm{P}$, Rufo-Smith C. Spinal surgery in patients with Parkinson's disease: construct failure and progressive deformity. Spine. 2004;29(18):2006-2012.

2. Benatru I, Vaugoyeau M, Azulay JP. Postural disorders in Parkinson's disease. Neurophysiol Clin. 2008;38(6):459-465.

3. Moon SH, Lee HM, Chun HJ, et al. Surgical outcome of lumbar fusion surgery in patients with Parkinson disease. $J$ Spinal Disord Tech. 2012;25(7):351-355.

4. Sapkas G, Lykomitros V, Soultanis K, Papadopoulos EC, Papadakis M. Spinal surgery in patients with Parkinson's disease: unsatisfactory results, failure and disappointment. Open Orthop J. 2014;8:264-267.

5. Ha Y, Oh JK, Smith JS, et al. Impact of movement disorders on management of spinal deformity in the elderly. Neurosurg. 2015;77(Suppl 4):S173-S185.

6. US Census Bureau: State and National Population Projections. http://www.census.gov/population/www/projections/ natproj.html and http://www.census.gov/popest/states/states.html. Accessed September 29, 2015.

7. Koller H, Acosta F, Zenner J, et al. Spinal surgery in patients with Parkinson's disease: experiences with the challenges posed by sagittal imbalance and the Parkinson's spine. Eur Spine J. 2010;19(10):1785-1794.

8. Peeraully T, Tan EK. Camptocormia in Parkinson's disease: dystonia or myopathy? Basal Ganglia. 2012;2(1):1-3.

9. van de Warrenburg BP, Cordivari C, Ryan AM, et al. The phenomenon of disproportionate antecollis in Parkinson's disease and multiple system atrophy. Mov Disord. 2007;22(16):2325-2331.

10. Di Matteo A, Fasano A, Squintani G, et al. Lateral trunk flexion in Parkinson's disease: EMG features disclose two different underlying pathophysiological mechanisms. J Neurol. 2011;258(5):740-745.

11. Peek AC, Quinn N, Casey AT, Etherington G. Thoracolumbar spinal fixation for camptocormia in Parkinson's disease. J Neurol Neurosurg Psychiatry. 2009;80(11):12751278.

12. Wadia PM, Tan G, Munhoz RP, Fox SH, Lewis SJ, Lang AE. Surgical correction of kyphosis in patients with camptocormia due to Parkinson's disease: a retrospective evaluation. J Neurol Neurosurg Psych. 2011;82:364368 .

13. Djaldetti R, Mosberg-Galili R, Sroka H, Merims D, Melamed E. Camptocormia (bent spine) in patients with
Parkinson's disease - characterization and possible pathogenesis of an unusual phenomenon. Mov Disord. 1999;14(13):443447.

14. Ashour R, Jankovic J. Joint and skeletal deformities in Parkinson's disease, multiple system atrophy, and progressive supranuclear palsy. Mov Disord. 2006;21(11):18561863.

15. Gerling MC, Bohlman HH. Dropped head deformity due to cervical myopathy: surgical treatment outcomes and complications spanning twenty years. Spine. 2008;33(20):E739E745.

16. Margraf NG, Wrede A, Rohr A, et al. Camptocormia in idiopathic Parkinson's disease: a focal myopathy of the paravertebral muscles. Mov Disord. 2010;25(5):542-551.

17. Bloem BR, Beckley DJ, van Dijk JG. Are automatic postural responses in patients with Parkinson's disease abnormal due to their stooped posture? Exp Brain Res. 1999;124(4):481488.

18. Bonanni L, Thomas A, Varanese S, Scorrano V, Onofrj M. Botulinum toxin treatment of lateral axial dystonia in Parkinsonism. Mov Disord. 2007;22(14):2097-2103.

19. Capelle HH, Schrader C, Blahak C, et al. Deep brain stimulation for camptocormia in dystonia and Parkinson's disease. J Neurol. 2010;258:96-103.

20. Uzawa A, Mori M, Kojima S, et al. Dopamine agonistinduced antecollis in Parkinson's disease. Mov Disord. 2009;24(16):2408-2411.

21. Baik, JS, Kim, JY, Park, JH, Han SW, Park JH, Lee MS. Scoliosis in patients with Parkinson's disease. J Clin Neurol. 2009;5(2):91-94.

22. Duvoisin RC, Marsden CD. Note on the scoliosis of parkinsonism. J Neurol Neurosurg Psychiatry. 1975;38(8):787793.

23. Marsden CD, Duvoisin R. Scoliosis and Parkinson's disease. Arch Neurol. 1980;37(4):253-254.

24. Vanderpool DW, James JI, Wynne-Davies R. Scoliosis in the elderly. J Bone Joint Surg Am. 1969;51(3):446-455.

25. Schroeder JE, Hughes A, Sama A, et al. Lumbar spine surgery in patients with Parkinson disease. J Bone Joint Surg Am. 2015;97(20):1661-1666.

26. Fujimoto K. Dropped head in Parkinson's disease. $J$ Neurol. 2006;253(Suppl 7):VII21-VII26.

27. Jorens P, Eycken M, Parizel G, Martin JJ. Antecollis in parkinsonism. Lancet. 1989;1(8650):1320-1321.

28. Yamada H, Katayama Y, Yamamoto T. Parkinson's disease and dropped head. Rinsho Shinkeigaku. 2003;43:955.

29. Umemura A, Oka Y, Ohkita K, Yamawaki T, Yamada K. Effect of subthalamic deep brain stimulation on postural abnormality in Parkinson disease. J Neurosurg. 2010;112(6):12831288.

30. Pereira EAC, Wilson-MacDonald J, Green AL, Aziz TZ, Cadoux-Hudson TAD. Posterior occipitocervical instrumented fusion for dropped head syndrome after deep brain stimulation. J Clin Neurosci. 2010;17(4):541-542.

31. Hellmann MA, Djaldetti R, Israel Z, Melamed E. Effect of deep brain subthalamic stimulation on camptocormia and postural abnormalities in idiopathic Parkinson's disease. Mov Disord. 2006;21(11):2008-2010.

32. Sako W, Nishio M, Maruo T, et al. Subthalamic nucleus deep brain stimulation for camptocormia associated 
with Parkinson's disease. Mov Disord. 2009;24(7):10761079 .

33. Wunderlich S, Csoti I, Reiners K, et al. Camptocormia in Parkinson's disease mimicked by focal myositis of the paraspinal muscles. Mov Disord. 2002;17(3):598-600.

34. Tassorelli C, Furnari A, Buscone S, et al. Pisa syndrome in Parkinson's disease: clinical, electromyographic, and radiological characterization. Mov Disord. 2012;27(2):227235.

35. Bhattacharya KF, Giannakikou I, Munroe N, Chaudhuri KR. Primary anticholinergic-responsive Pisa syndrome. Mov Disord. 2000;15(6):1285-1287.

36. Villarejo A, Camacho A, García-Ramos R, et al. Cholinergic-dopaminergic imbalance in Pisa syndrome. Clin Neuropharmacol. 2003;26(3):119-121.

37. Yokochi F. Lateral flexion in Parkinson's disease and Pisa syndrome. J Neurol. 2006;253(Suppl 7):VII17-VII20.

38. Cannas A, Solla P, Floris G, et al. Reversible Pisa syndrome in patients with Parkinson's disease on dopaminergic therapy. J Neurol. 2009;256(3):390-385.

39. Upadhyaya CD, Starr PA, Mummaneni PV. Spinal deformity and Parkinson disease: a treatment algorithm. Neurosurg Focus. 2010;28(3):E5.

40. Palumbo MA, Shah KN, Eberson CP, Hart RA,
Daniels AH. Outrigger rod technique for supplemental support of posterior spinal arthrodesis. Spine J. 2015;15(6):1409-1414.

Disclosures and COI: No funding was obtained in support of this work.

Corresponding Author: Alan H. Daniels, MD, Department of Orthopaedic Surgery, Warren Alpert Medical School of Brown University, 100 Butler Drive, Providence, RI 02906. Phone: (401) 330-1420; Fax: (401) 330-1495; Email: Alan_Daniels@Brown.edu.

Published 30 March 2018

This manuscript is generously published free of charge by ISASS, the International Society for the Advancement of Spine Surgery. Copyright (C) 2018 ISASS. To see more or order reprints or permissions, see http://ijssurgery.com. 